\title{
Clinical investigation of the safety and efficacy of a cervical intraepithelial neoplasia treatment using a hyperthermia device that uses heat induced by alternating magnetic fields
}

\author{
KOJI KOIZUMI ${ }^{1}$, TORU FUJIOKA ${ }^{2}$, TOSHIAKI YASUOKA ${ }^{2}$, AYA INOUE ${ }^{2}$, YUKA UCHIKURA ${ }^{2}$, \\ HIROKI TANAKA ${ }^{2}$, KATSUKO TAKAGI ${ }^{2}$, MIKI MORI ${ }^{2}$, MASAE KOIZUMI ${ }^{2}$, HISASHI HASHIMOTO ${ }^{2}$, \\ TAKASHI MATSUMOTO ${ }^{2}$, YUKO MATSUBARA ${ }^{2}$, KEIICHI MATSUBARA $^{2}$ and AKIHIRO NAWA ${ }^{2}$ \\ ${ }^{1}$ Bara-no-izumi Women's Clinic, Matsuyama, Ehime 790-0941; ${ }^{2}$ Department of Obstetrics and \\ Gynecology, Ehime University, Graduate School of Medicine, Toon, Ehime 791-0295, Japan
}

Received April 23,2015; Accepted May 13, 2016

DOI: $10.3892 / \mathrm{mco} .2016 .929$

\begin{abstract}
Multiple techniques have been used for the conservative treatment of high-grade cervical intraepithelial neoplasia (HG-CIN) in women of fertile age. Conization has been associated with stenosis of the cervix and a decrease in cervical mucus secretion, in addition to the increase in the risk of cervical canal shortening and problems during the perinatal period, including premature birth and premature rupture of membranes. Although the laser transpiration technique does not cause shortening of the cervical canal, it is associated with the recurrent risk of deep residual disease. The present study aimed to investigate the therapeutic safety and efficacy of the therapy performed using the transaction magnetic field induction heating device, AMTC400, in fertile patients with HG-CIN (excluding carcinoma in situ). Four premenopausal patients with CIN3 and high-risk human papilloma virus (HPV)-positive were treated using an AMTC400. Chronological colposcopic findings, high-risk HPV, final histological findings with conization and follow-up data were evaluated. All the treatments were successfully performed on the in-patients without anesthesia. Intra- and postoperative complications included minor pain and bleeding in all cases. Two of the cases (50\%) were high-risk HPV-negative following the treatments. All cases exhibited a change in the observed color (to white), and subsequent epithelization following treatment. Although cytological analysis at 5 weeks following the treatment confirmed the cases were negative for intraepithelial lesions and malignancies, a definitive histology with conization 6 weeks following the treatment confirmed CIN1 and koilocytosis in all cases. The assessment
\end{abstract}

Correspondence to: Dr Toru Fujioka, Department of Obstetrics and Gynecology, Ehime University, Graduate School of Medicine, Shitsukawa, Toon, Ehime 791-0295, Japan

E-mail: fujioka@m.ehime-u.ac.jp

Key words: hyperthermia device, cervical intraepithelial neoplasia, alternating magnetic field, conservative treatment of treatment effectiveness was determined as a moderate improvement in all cases. In conclusion, thermotherapy applied using AMTC400 represented a safe and effective treatment for HG-CIN in women of fertile age. However, additional improvements associated with the site of puncture needles are required. Further studies are required to confirm the long-term efficacy and reproductive outcomes.

\section{Introduction}

Dysplasia that is seen on a biopsy of the cervix is called cervical intraepithelial neoplasia (CIN). It is grouped into three categories: CIN I (mild dysplasia), CIN II (moderate to marked dysplasia) and CIN III (severe dysplasia to carcinoma in situ). Treating high-grade (HG)-CIN reduces the incidence and mortality caused by invasive cervical cancer in women with these lesions (1-3). Women treated for CIN are of reproductive age (mean age of approximately 30 years), although the disorder may also arise in much younger women $(4,5)$.

Multiple techniques are available for the conservative treatment of HG-CIN in women of fertile age (6-9). These include ablative methods (e.g., cryotherapy, laser vaporization and cold coagulation), with satisfactory colposcopic examination results in women for whom invasion has been ruled out and excisional methods [e.g., the loop electrosurgical excision procedure (LEEP), laser conization and cold-knife conization] for women whose colposcopic examinations are unsatisfactory. Hysterectomy is ruled out as a primary therapy option for HG-CIN woman of fertile age. Cold knife conization, laser ablation, laser conization and large loop excision of the transformation zone (LLETZ; an alternative term for LEEP) are all conservative treatment methods used to remove or destroy transformation zones containing abnormal cells and to preserve cervical function at the same time $(10,11)$. Cold knife conization was closely associated with preterm delivery, low birth weight and cesarean sections. LLETZ was also closely associated with preterm delivery, low birth weight and premature rupture of the membranes. All excisional procedures to treat CIN are associated with a small, but real increase in the risk of pregnancy-related morbidity (12-15). Although no 
markedly increased risks for obstetric outcomes following laser ablation were detected (14), the recurrence rate of CIN3 in the first year following treatment was reported as $22.6 \%$ in a different study (16). Although the reported primary cure rate of conservative treatment for HG-CIN exceeds 95\%, a significant number of patients show persistent or recurring disease during the follow-ups $(6-9,17,18)$. Appropriate management of women with HG-CIN is a critical component of cervical cancer prevention. Improper management may increase the risk of both cervical cancer and complications from over-treatment, such as preterm delivery $(14,19)$.

Photodynamic therapy (PDT) uses photosensitizing agents, oxygen and light to create a photochemical reaction that selectively destroys cancer cells. Photosensitizing agents are drugs, administered in the body, which become concentrated in cancer cells and are activated only when light of a certain wavelength is directed to the area affected by the cancer. The photodynamic reactions between the photosensitizing agent and light and oxygen kill the cancer cells (20). These patients may find all parts of their body sensitive to light, and are advised to take precautions to protect themselves from light for the necessary length of time, ranging from days to weeks, depending on the photosensitizing drug used. Patients are also advised to avoid intense direct sunshine for approximately 6 months following PDT. Due to patient burdens, this is not the standard treatment for HG-CIN in women of fertile age.

On the other hand, thermal ablation is a new heat-based cancer therapy that kills tumor cells by means of heat induction in a magnetic metal subjected to alternating magnetic fields. This technique has recently been put into practice, and has proven to be effective in rat subcutaneous tumor and liver tumor models (21). Applying this principle to treat CIN, including cervical dysplasia and cervical carcinoma in situ, eliminates the need for trachelectomy while exerting therapeutic effects on deep foci. Thus, this treatment method offers the advantages of both conization and laser vaporization. However, its safety and efficacy have yet to be investigated, even with respect to the development of associated medical devices.

Developed by AdMeTech Co., Ltd. (Matsuyama, Japan) to treat CIN, the AMTC400 is a hyperthermia-inducing device that harnesses the principle of heat induction by alternating magnetic fields to convert magnetic energy into heat, applying alternating magnetic fields to a magnetic needle inserted into the uterine cervix via the applicator tip. The device achieves precise control of the temperature of the treated area, maintaining temperatures at $\sim 60^{\circ} \mathrm{C}$ without passing a high-frequency electric current through the body, ensuring that deep-lying areas are also heated. Classified as high-temperature hyperthermia, the treatment method used with this device is one of several possible hyperthermia therapies.

The aim of the present study was to investigate the therapeutic safety and efficacy of the therapy performed by the transaction magnetic field induction heating device, AMTC400, in fertile patients with HG-CIN (excluding carcinoma in situ).

\section{Patients and methods}

The present clinical study was undertaken at the authors' institution between April 2012 and March 2013 in compli- ance with the following protocol. Women of ages ranging from 20 to 39 years were investigated. The inclusion criteria were as follows: A diagnosis of CIN3 (excluding carcinoma in situ) based on cytology, histology and colposcopy; location of the lesion in a visible area; a positive result from a high-risk HPV test; and consent to the treatment method in question.

The lesions were treated in the uterine cervix of the patients using AMTC400, the transaction magnetic field induction heating device developed by AdMeTech Co., Ltd., that uses heat induced by alternating magnetic fields. Cytological and high-risk HPV tests were performed approximately 5 weeks afterwards, with subsequent conization using an ultrasonic scalpel for a histopathological examination at 6 weeks following treatment of the lesions.

An uncontrolled open-label study design was selected, since Japan currently lacks an established standard therapy for the condition treated in the present investigation that retains the uterine cervix. Additionally, since this was an exploratory clinical trial to confirm the safety and efficacy of the treatment, the target number of patients was set at six, so that the probability of detecting at least one adverse event whose incidence rate was $25 \%$ would be $80 \%$. The clinical trial described above was undertaken with the approval of the ethics committee at Ehime University, Graduate School of Medicine (Ehime, Japan).

Composition of the device, AMTC400. The medical device in question is composed of a main unit that generates high-frequency magnetic fields and heating needles that are inserted into the affected areas (Figs. 1 and 2). The heating needles convert alternating magnetic fields into heat. Two types of needles were used for this trial: One combined with a temperature-measuring thermocouple for measuring temperature and heating, and another that simply emits heat (Fig. 2).

Treatment procedure. The treatment procedure was to puncture the lesion with heating needles under colposcopy, and subsequently to insert the tip of the applicator on the main unit of the device into the vagina to apply alternating magnetic fields to the heating needles. The resulting heat emitted by the heating needles denatures the surrounding lesion. A detailed description of the procedure was as follows: i) The switch on the control panel on the main unit of the device was activated to confirm that the device was functioning. ii) The investigator or the subinvestigator explained to the patient that hyperthermia using heat induced by alternating magnetic fields would begin, and instructed the patient to sit in an examining chair in a lithotomy position. iii) The vaginal canal was disinfected with a plastic Cusco speculum, the affected area was subsequently located, and images were captured under colposcopy. iv) The located affected area was punctured with the appropriate number of heating needles or temperature-measuring/heating needles using Kocher clamps with a space of $5 \mathrm{~mm}$ between each needle, since the effect of heat induction was to reach up to $5 \mathrm{~mm}$ wide in the preliminary study (data not shown). If the lesion was punctured with temperature-measuring/heating needles, the thermocouple connector of the thermocouple signal cable was connected to the arm on the main unit of the device afterwards. v) Two temperature-measuring/heating needles were used to control the device. For the first three patients, 


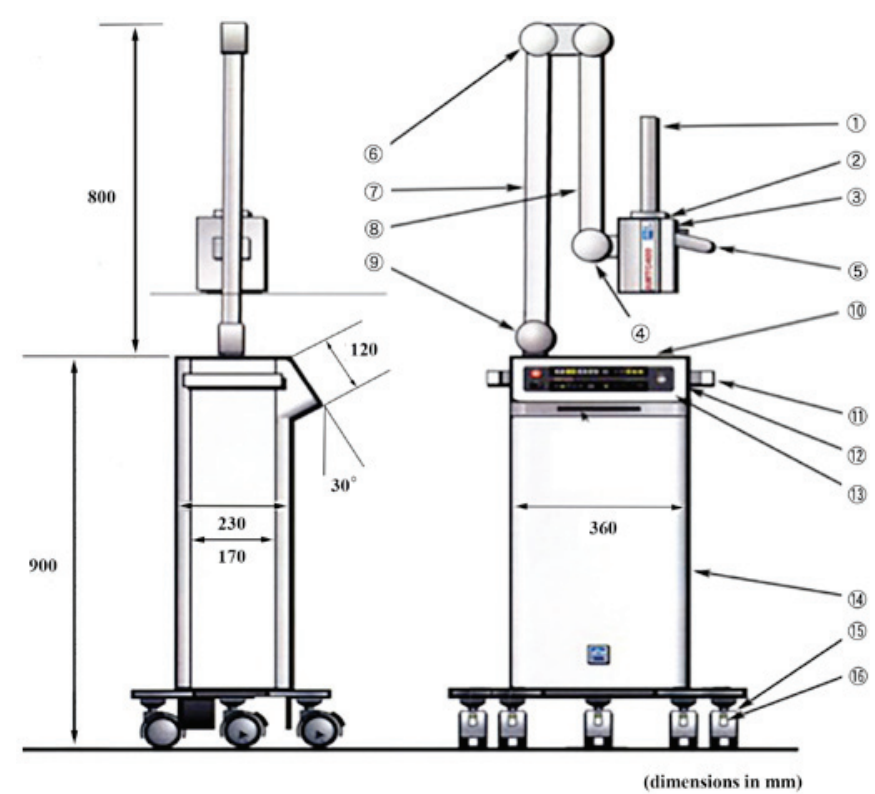

Figure 1. Structure of the main unit of the device and part names. 1, applicator: This is the part inserted into a body cavity in which alternating magnetic fields are generated; 2, resonant box: The applicator is attached to the end of the resonance circuit; 3 ; resonant box cover: The box features a sliding mechanism that moves backwards and forwards over the resonant box, serving as a cover; 4, Knob used to lock the resonant box cover: This knob secures the sliding mechanism of the resonant box; 5, Grip: This is the grip grasped when moving the resonant box forward or backward; 6 , first arm-securing knob: This knob secures the arm in place to keep it from moving up or down; 7 , arm: This mechanism supports the resonant box; used to move the box up and down and right and left; 8, thermosensor connector: Heating needles with thermosensors are connected here; 9 , second arm-securing knob: This knob secures the arm in place to keep it from moving right or left; 10, tray: The tray can be used for temporary placement of thermosensors; 11, side handle: This handle is used when moving the entire device; 12 , card slot: This slot accepts MMC or SD cards; 13, control panel: The control panel is used to control the device, including starting or stopping treatment; 14, power control unit: This is the device component used to control electric power and the microcomputer; 15, casters: These casters make it easy to move the device with braking levers; 16 , caster braking levers: Use these levers to secure the casters and to prevent the device from moving.

only temperature-measuring/heating needles were used to ensure that all inserted heating needles emitted heat equally. If three or more temperature-measuring/heating needles were used, the temperature of each needle was monitored and recorded separately using a battery-operated $5 \mathrm{~V}$ direct current temperature-measuring device supplied by the sponsor (AdMeTech Co., Ltd.). vi) The applicator cover and the probe cover were attached to the applicator on the main unit of the device, and this was inserted into the vagina until it touched the heating needles. vii) The 'Start Treatment' button on the control panel on the device was pushed to start the device. Once treatment began, the heating needles began to heat. Their temperature was automatically maintained at $60 \pm 5^{\circ} \mathrm{C}$, and the temperature of the affected area was $\sim 55 \pm 5^{\circ} \mathrm{C}$. viii) Since the patient's movements may increase the distance between the applicator and the heating needles, the heat emitted by the heating needles was reduced during treatment, and the investigator or subinvestigator was required to keep his or her hand on the applicator at all times, remaining aware of any sudden movements and keeping the heating needles and the applicator in contact. ix) If all devices operated normally, the
A

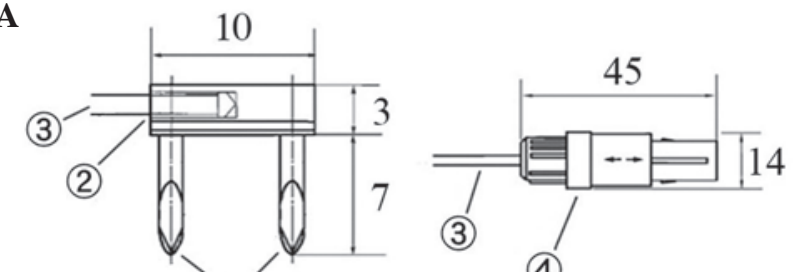

(1)

B

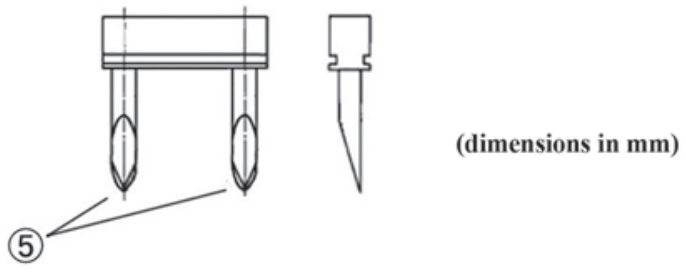

Figure 2. Structure and part names of the two types of needles. (A) Temperature-measuring/heating needle. 1, heating needles: Heating elements inserted into affected areas; 2 , thermocouple: Thermosensor used to measure temperatures; 3 , thermocouple signal cable: Coated signal cable for thermosensors; 4, connector: Used to connect thermosensors to the main unit of the device. (B) Heating needle. 5, heating needles: Heating elements inserted into the affected areas.

output of the high-frequency magnetic fields automatically ceased within $10 \mathrm{~min}$, and the heating needles stopped emitting heat. $\mathrm{x}$ ) The applicator was removed from the vagina, all heating and temperature-measuring/heating needles were removed, and it was made certain that bleeding had been arrested. The affected area was disinfected and images were captured. xi) The Cusco speculum was removed to conclude the procedure. xii) A final check was performed to ensure the absence of bleeding 30-60 min following the end of the treatment.

Endpoints for efficacy. Specimens excised during conization 6 weeks following the treatment were histologically examined and assessed on the following 6-point scale: Cure, no dysplasia detected; moderate improvement, CIN1 detected; mild improvement, CIN2 detected; No change, CIN3 (with no carcinoma in situ) detected; progression, carcinoma in situ detected; aggravation, minimally invasive squamous cell carcinoma or squamous cell carcinoma detected.

\section{Results}

The treatment was administered to four patients who provided their consent (Table I). The lesions were located and punctured with heating needles under colposcopy (Fig. 3). No anesthesia was required; intraoperative pain and heat sensations were mild and required no additional intervention. Postoperative bleeding following the removal of the needles was minimal and stopped by gauze compression (Fig. 4).

With respect to chronological changes observed via colposcopy, the vaginal portion of the cervix had turned white by postoperative day 3 , and exhibited erosion with a partial white coating by postoperative day 10 in all cases. At postoperative day 31 , the erosion had diminished, with overall epithelialization under way (Fig. 5).

No clear symptoms other than minor bleeding were reported following the treatment. At approximately 4 weeks 
Table I. Patient characteristics, high-risk HPV test results, histopathological results following conization and efficacy assessment.

\begin{tabular}{|c|c|c|c|c|c|c|}
\hline Case & Age & $\begin{array}{c}\text { Pregnancy/ } \\
\text { delivery } \\
\text { history }^{\mathrm{a}}\end{array}$ & High-risk HPV & $\begin{array}{l}\text { Cytology } \\
\text { (5 weeks } \\
\text { following } \\
\text { treatment) }\end{array}$ & $\begin{array}{l}\text { Histopathology } \\
\text { (conization, } \\
6 \text { weeks } \\
\text { following } \\
\text { treatment) }\end{array}$ & Efficacy assessment \\
\hline 1 & 36 & G0P0 & Type $16 \rightarrow$ type 16 & NILM & CIN1 & Moderate improvement \\
\hline 2 & 34 & G1P1 & Type $52 \rightarrow$ type 52 & NILM & CIN1 & Moderate improvement \\
\hline 3 & 32 & G1P1 & Type $16,58 \rightarrow$ negative & NILM & CIN1 & Moderate improvement \\
\hline 4 & 35 & G3P1 & Type $16 \rightarrow$ negative & NILM & CIN1 & Moderate improvement \\
\hline
\end{tabular}

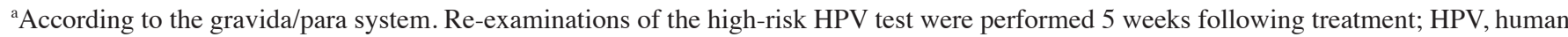
papilloma virus; NILM, negative for intraepithelial lesions and malignancies; CIN1, cervical intraepithelial neoplasia 1.

A

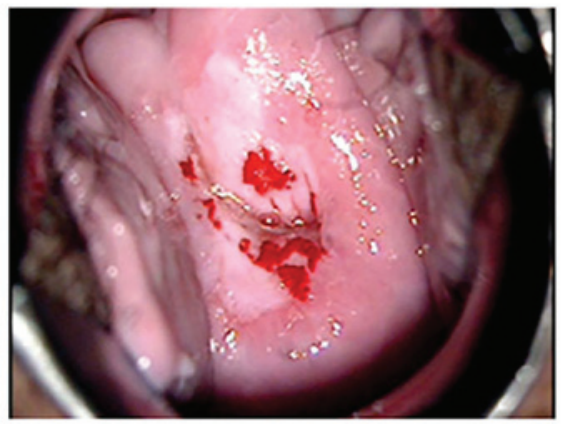

B

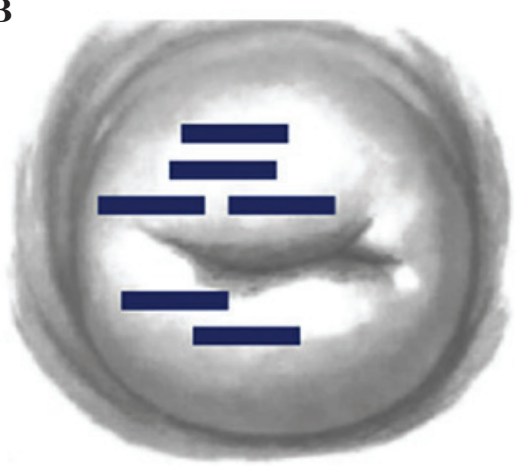

Figure 3. Colposcopic findings prior to treatment, and a pattern diagram of the inserted heating needles (patient 4). (A) Colposcopic findings prior to the treatment: Colposcopy performed prior to the insertion of the heating needles revealed an abnormality (white epithelium). (B) Pattern diagram of the inserted heating needles: Heating needles were inserted in the area where the abnormality was found.
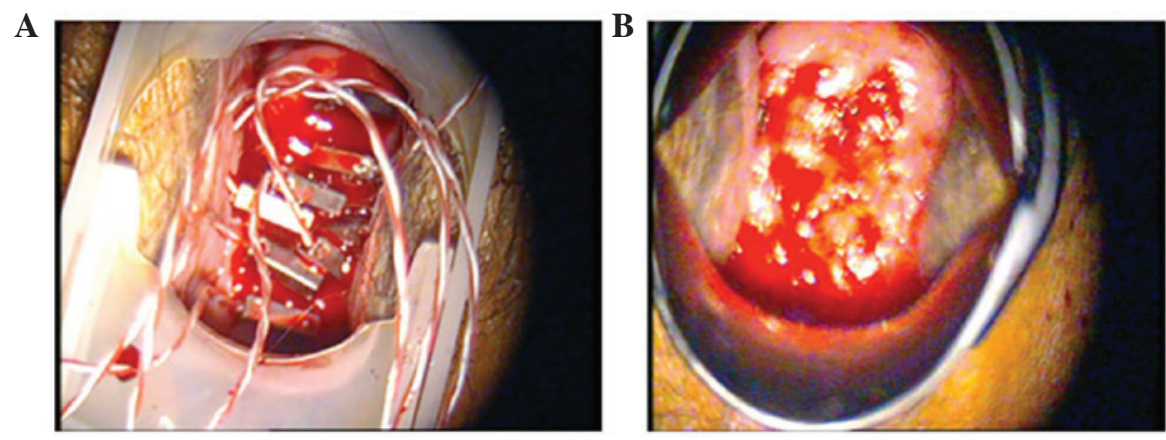

Figure 4. Vaginal portion of the cervix after treatment (patient 2). (A) Vaginal portion of the cervix immediately following hyperthermia: A total of eight temperature-measuring/heating needles or heating needles were inserted into the area where the abnormality was found. (B) Following removal of the needles: minor bleeding ensued, although this was arrested by gauze compression.

following the treatment, high-risk HPVs had disappeared from two of the four patients (Table I).

A colposcopy performed approximately 5 weeks after treatment revealed no apparent abnormal findings in any of the patients. The results of the cytology were negative for intraepithelial lesions and malignancies (NILM) in all four patients. However, histopathological examination of the conization specimens revealed koilocytosis in the surface layers, with atypical cells in parts of the lower third of the epithelium in all four patients, leading to a diagnosis of CIN1 (Fig. 6). The efficacy assessment was moderate improvement in all four patients (Table I).

With respect to safety analysis, one case had mild pain following the treatment, although no serious adverse events associated with the treatment were observed (Table II). Device malfunctions or defects emerged with two patients. The first instance involved a pinhole on the probe cover discovered following treatment. Examination of the affected area revealed no abnormalities, and the patient was unaffected. As a countermeasure, the use of the probe covers was 
Table II. Safety analysis.

Occasion

of defect

malfunction

Occurrence

Number (\%)

1

Occurrences of related serious adverse events

Number of occurrences (incidence)

2

Occurrences of malfunctions or defects

Number of occurrences (incidence)

Description of malfunction or defect

A pinhole was found on the probe cover

The needle part of a temperature-measuring/heating needle was disconnected

$1(25.0 \%)$

A total of two occasions (1 and 2) of device malfunctions or defects emerged in treatments involving two of the patients. The patients were unaffected.

A

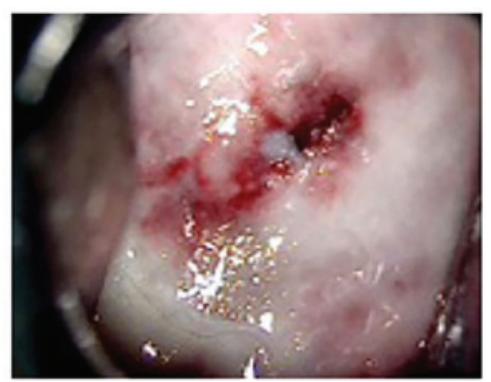

$\mathbf{B}$

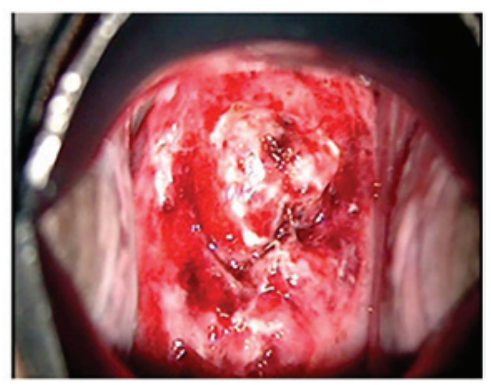

C

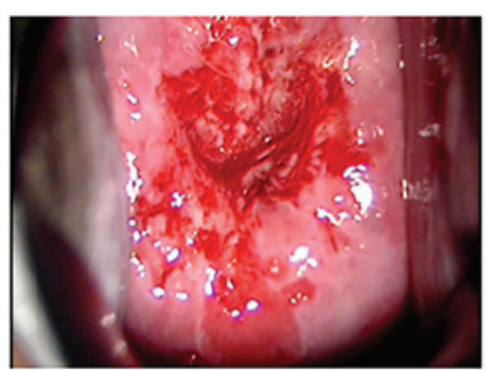

Figure 5. Vaginal portion of the cervix after hyperthermia (patient 2). (A) At 3 days later: Most of the vaginal portion of the cervix had turned white. (B) At 10 days later: the entire vaginal portion of the cervix exhibited erosion with a partial white coating. (C) At 31 days later: the area eroded had diminished, with overall epithelialization under way.

discontinued, and the enrolment of new patients was halted until a preventive measure had been implemented. The second instance involved disconnection of the needle portion of the temperature-measuring/heating needle during treatment

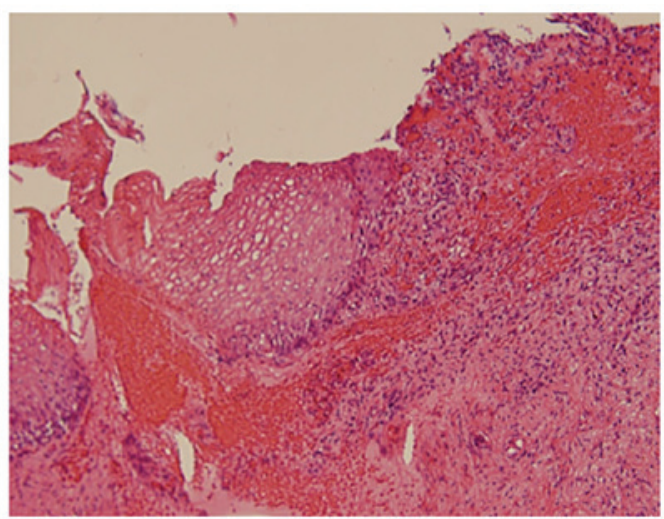

Figure 6. Histopathological examination following conization (patient 2) Colposcopy performed prior to conization revealed no apparent abnormalities; however, histopathological examination of the conization specimens revealed koilocytosis in the surface layer, with atypical cells in the lower third of the epithelium, leading to a diagnosis of CIN1. Original magnification, $\mathrm{x} 200$.

preparations. This issue arose prior to commencing the treatment, and a spare temperature-measuring/heating needle was used instead, treating the patient as planned, although without any effects on the patient. As a countermeasure, the use of the temperature-measuring/heating needles was discontinued and the enrolment of new patients was halted until a preventive measure had been implemented (Table II).

\section{Discussion}

To the best of our knowledge, this is the first time that a report has been published of studies undertaken on the basis of the principle applied in the present treatment method. The efficacy of treatment applied to treat HG-CIN with a hyperthermia by the transaction magnetic field induction heating device, AMTC400, in the present investigation was deemed to constitute a moderate improvement. Conization specimens from all four patients were found to have CIN1. Colposcopy performed prior to conization revealed no apparent abnormalities in any of the four patients, since they were all in the process of healing from the changes resulting from hyperthermia at 
Table III. Heating needle insertion sites and CIN1 sites.

\begin{tabular}{|c|c|c|c|c|c|c|c|c|}
\hline \multirow[b]{2}{*}{ Direction } & \multicolumn{2}{|c|}{ Case 1} & \multicolumn{2}{|c|}{ Case 2} & \multicolumn{2}{|c|}{ Case 3} & \multicolumn{2}{|c|}{ Case 4} \\
\hline & $\begin{array}{l}\text { Heating } \\
\text { needle } \\
\text { insertion }\end{array}$ & Dysplasia & $\begin{array}{l}\text { Heating } \\
\text { needle } \\
\text { insertion }\end{array}$ & Dysplasia & $\begin{array}{l}\text { Heating } \\
\text { needle } \\
\text { insertion }\end{array}$ & Dysplasia & $\begin{array}{l}\text { Heating } \\
\text { needle } \\
\text { insertion }\end{array}$ & Dysplasia \\
\hline 1 & - & - & + & - & + & - & + & - \\
\hline 2 & - & - & + & - & - & CIN1 & - & CIN1 \\
\hline 3 & - & - & - & - & - & CIN1 & - & - \\
\hline 4 & - & - & + & - & - & - & - & - \\
\hline 5 & + & - & + & - & + & - & - & - \\
\hline 6 & + & - & + & - & + & - & + & - \\
\hline 7 & + & - & + & - & + & - & + & - \\
\hline 8 & - & - & - & - & + & - & + & - \\
\hline 9 & - & - & - & CIN1 & - & - & + & - \\
\hline 10 & - & CIN1 & + & - & - & - & + & - \\
\hline 11 & - & CIN1 & + & - & + & - & + & - \\
\hline 12 & - & - & + & - & + & - & + & - \\
\hline
\end{tabular}

CIN1, cervical intraepithelial neoplasia 1.

A
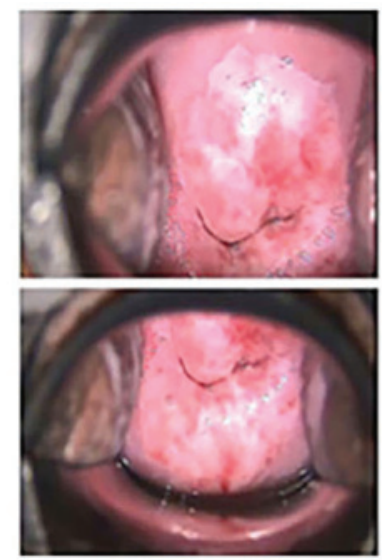

\section{B}

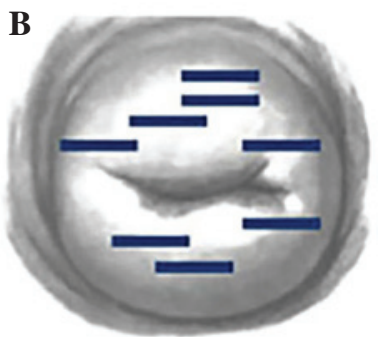

C

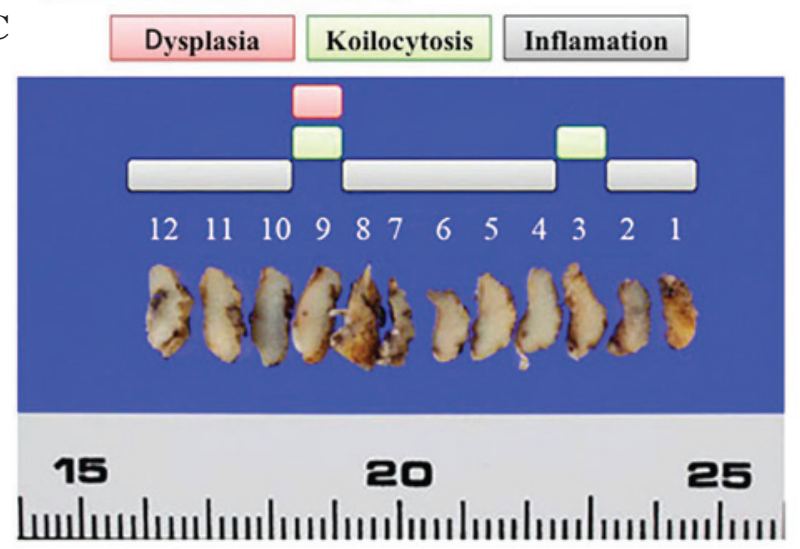

Figure 7. Colposcopic findings prior to treatment, with a pattern diagram of the inserted heating needles and histopathological findings following conization (patient 2). (A) Colposcopic findings prior to treatment: The epithelium appeared white over a wide portion of the vaginal area of the cervix, and exhibited a partial mosaic pattern. (B) Heating needles were inserted into the area where the abnormality had been located (bold lines). (C) Histopathological findings following conization. Inflammatory cells were identified throughout: Koilocytosis and dysplasia were revealed in slices from the 3 and 9 o'clock positions that had not been punctured with needles, since the abnormal colposcopic findings were undetectable or unclear. that time. Although the results of cytology were NILM for all four patients, histopathological examination of conization specimens revealed koilocytosis in the surface layers, with atypical cells in parts of the lower third of the epithelium in all four patients, leading to a diagnosis of CIN1. Comparing these areas with areas punctured with heating needles as part of the treatment revealed that the former had not been punctured with needles, since the colposcopy performed prior to the treatment had resulted in an assessment that abnormalities were absent (Fig. 7 and Table III). In other words, the heating applied may have been inadequate, leading to CIN1 being present prior to treatment, which persisted following treatment. Possible future improvements in this procedure would involve puncturing the entire vaginal area of the cervix with heating needles without relying on the findings from colposcopy; this may lead to improved therapeutic effects.

As for the analysis of treatment safety, no serious adverse events were observed other than mild pain and bleeding. Two instances of device malfunctions or defects emerged, but neither was detrimental to the patients, and both were addressed and later rectified.

In conclusion, we consider that the treatment method explored in the present study may prove beneficial to younger patients who wish to preserve their fertility. The method does not involve trachelectomy, despite room for improvement with respect to the areas punctured with heating needles. Since it requires no anesthesia and the burden on the patients is small, our consideration is that this treatment may be provided on an outpatient basis. The method requires improvements with respect to the areas punctured with heating needles, and future studies should accumulate additional case studies.

In conclusion, the thermotherapy administered with the transaction magnetic field induction heating device, AMTC400, appears to be safe and effective in terms of treating HG-CIN in women of fertile age, although further 
improvements are required with respect to puncture needle sites. Further studies are also required to confirm long-term efficacy and reproductive outcomes.

\section{References}

1. McCredie MR, Sharples KJ, Paul C, Baranyai J, Medley G, Jones RW and Skegg DC: Natural history of cervical neoplasia and risk of invasive cancer in women with cervical intraepithelial neoplasia 3: A retrospective cohort study. Lancet Oncol 9: 425-434, 2008.

2. Soutter WP, Sasieni P and Panoskaltsis T: Long-term risk of invasive cervical cancer after treatment of squamous cervical intraepithelial neoplasia. Int J Cancer 118: 2048-2055, 2006.

3. Strander B, Andersson-Ellström A, Milsom I and Sparén P: Long term risk of invasive cancer after treatment for cervical intraepithelial neoplasia grade 3: Population based cohort study. BMJ 335: 1077, 2007.

4. Herbert A: Cervical screening in England and Wales: Its effect has been underestimated. Cytopathology 11: 471-479, 2000.

5. Paraskevaidis E, Kitchener HC, Miller ID, Mann E, Jandial L and Fisher PM: A population-based study of microinvasive disease of the cervix - a colposcopic and cytologic analysis. Gynecol Oncol 45: 9-12, 1992.

6. Fallani MG, Penna C, Fambrini M and Marchionni M: Laser CO2 vaporization for high-grade cervical intraepithelial neoplasia: A long-term follow-up series. Gynecol Oncol 91: 130-133, 2003.

7. Mathevet P, Chemali E, Roy M and Dargent D: Long-term outcome of a randomized study comparing three techniques of conization: Cold knife, laser, and LEEP. Eur J Obstet Gynecol Reprod Biol 106: 214-218, 2003.

8. Ueda M, Ueki K, Kanemura M, Izuma S, Yamaguchi $H$ Nishiyama K, Tanaka Y, Terai Y and Ueki M: Diagnostic and therapeutic laser conization for cervical intraepithelial neoplasia. Gynecol Oncol 101: 143-146, 2006.

9. van Hamont D, van Ham MA, Struik-van der Zanden PH, Keijser KG, Bulten J, Melchers WJ and Massuger LF: Long-term follow-up after large-loop excision of the transformation zone: Evaluation of 22 years treatment of high-grade cervical intraepithelial neoplasia. Int J Gynecol Cancer 16: 615-619, 2006.

10. Kitchener HC, Cruickshank ME and Farmery E: The 1993 British society for colposcopy and cervical pathology/national coordinating network united kingdom colposcopy survey. Comparison with 1988 and the response to introduction of guidelines. Br J Obstet Gynaecol 102: 549-552, 1995.
11. Prendiville W, Cullimore J and Norman S: Large loop excision of the transformation zone (LLETZ). A new method of management for women with cervical intraepithelial neoplasia. Br J Obstet Gynaecol 96: 1054-1060, 1989.

12. Arbyn M, Kyrgiou M, Simoens C, Raifu AO, Koliopoulos G, Martin-Hirsch P, Prendiville W and Paraskevaidis E: Perinatal mortality and other severe adverse pregnancy outcomes associated with treatment of cervical intraepithelial neoplasia: Meta-analysis. BMJ 337: a1284, 2008.

13. Bevis KS and Biggio JR: Cervical conization and the risk of preterm delivery. Am J Obstet Gynecol 205: 19-27, 2011.

14. Kyrgiou M, Koliopoulos G, Martin-Hirsch P, Arbyn M, Prendiville W and Paraskevaidis E: Obstetric outcomes after conservative treatment for intraepithelial or early invasive cervical lesions: Systematic review and meta-analysis. Lancet 367: 489-498, 2006.

15. Sadler L, Saftlas A, Wang W, Exeter M, Whittaker J and McCowan L: Treatment for cervical intraepithelial neoplasia and risk of preterm delivery. JAMA 291: 2100-2106, 2004.

16. Inaba K, Nagasaka K, Kawana K, Arimoto T, Matsumoto $Y$, Tsuruga T, Mori-Uchino M, Miura S, Sone K, Oda K, et al: High-risk human papillomavirus correlates with recurrence after laser ablation for treatment of patients with cervical intraepithelial neoplasia 3: A long-term follow-up retrospective study. J Obstet Gynaecol Res 40: 554-560, 2014.

17. Ghaem-Maghami S, Sagi S, Majeed G and Soutter WP: Incomplete excision of cervical intraepithelial neoplasia and risk of treatment failure: A meta-analysis. Lancet Oncol 8: 985-993, 2007.

18. Skjeldestad FE, Hagen B, Lie AK and Isaksen C: Residual and recurrent disease after laser conization for cervical intraepithelial neoplasia. Obstet Gynecol 90: 428-433, 1997.

19. Insinga RP, Glass AG and Rush BB: Diagnoses and outcomes in cervical cancer screening: A population-based study. Am J Obstet Gynecol 191: 105-113, 2004.

20. Chen J, Keltner L, Christophersen J, Zheng F, Krouse M, Singhal A and Wang SS: New technology for deep light distribution in tissue for phototherapy. Cancer J 8: 154-163, 2002.

21. Kikkawa H: Anti-tumor effects of induced hyperthermia using a $\mathrm{MgFe} 2 \mathrm{O} 4$ needle in rat tumor models. Ehime Igaku 24: 156-164, 2005. 